\title{
A condition for robust stabilizability
}

\author{
Huibert KWAKERNAAK \\ Department of Applied Mathematics. Twente University of Tech- \\ nology, P.O. Box 217, 7500 AE Enschede. The Netherlands
}

Received 10 August 1981

Revised 31 March 1982

\begin{abstract}
A family of linear time-invariant finite-dimensional plants is called robustly stabilizable if there exists a single controller that stabilizes each plant in the family. A condition for robust stabilizability is given that requires the plant transmission zeros to remain in the left-half complex plane and moreover imposes a restriction on the high-frequency behavior of the plant transfer matrix.
\end{abstract}

Keywords: Robustness, Stabilizability, High-gain feedback, Linear optimal control, Desensitization.

\section{Introduction}

Currently there is considerable interest in the design of robust control systems, that is, control systems that retain their properties under plant perturbations. Some notable contributions in this area are the work of Wonham [1], Davison (see e.g. [2]), Safonov (see e.g. [3]) and Doyle [4,5] Wonham and Davison studied control systems that retain disturbance rejecting and tracking properties under plant perturbations. Safonov investigated the robustness of linear optimal state feedback control systems, while Doyle developed a criterion for the stability robustness of closed-loop systems.

A crucial aspect of a control system is its stability. This property above all should be preserved under plant perturbations. We therefore investigate in this paper robust stabilizability. This property is enjoyed by a family of plants $\mathscr{P}$ if there

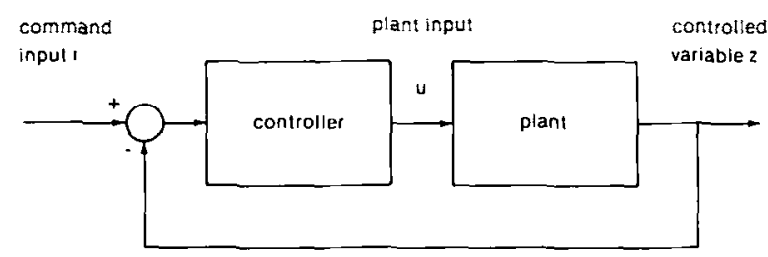

Fig. 1. Control system configuration. exists a single fixed controller such as in the configuration of Figure 1 that stabilizes the closed-loop system when the plant is any member of the family os. The problem of finding such a controller, termed the simultaneous stabilization problem, has been considered by Saeks and Murray [6]. As they point out, however, the solution they present is mathematical in nature and not intended for computational implementation. In the present paper, a plausible and directly verifiable (sufficient) condition for the existence of a solution to the simultaneous stabilization problem is obtained.

In this paper, all the plants in the family $\mathscr{P}$ to be considered are assumed to be linear, time-invariant and finite-dimensional, with the same number of inputs as outputs $p$. The transfer matrix of any plant in $P$ will be denoted as $H(s)$. One special member of $\mathscr{P}$ will be referred to as the nominal plant; its transfer matrix is $H_{0}(s)$. The loop gain of the control system, which will always be assumed to be in the configuration of Figure 1, is $L(s):=H(s) G(s)$, where $G$ is the transfer matrix of the controller (which is also assumed to be linear and time-invariant). The control system sensitivity matrix is $S(s):=[I+L(s)]^{-1}$. The corresponding quantities for the nominal plant are respectively $L_{0}$ and $S_{0}$.

\section{Desensitization}

Recently, work has been done on the question how to achieve sensitivity reduction of multivariable closed-loop systems. Zames [7] showed that for plants that have no transmission zeros in the right-half complex plane arbitrarily small sensitivity may be obtained. Using quite different techniques, Kimura [8] obtained comparable results.

In the next section it will be seen that such a system - naturally - also possesses a degree of robustness. Before proceeding to this topic we state and prove in the present section a result that is analogous to those of Zames and Kimura but put in a form in which it is needed in the sequel. 
Theorem 1. Suppose that the nominal plant is stabilizable, detectable and invertible and has all its transmission zeros in the open left-half complex plane. Then for each $\varepsilon>0$ there exists an $\omega_{0}>0$ and $a \rho_{0}>0$ such that for all $\rho_{0} \geqslant \rho_{0}$ a stabilizing controller may be found with the property that the corresponding nominal sensitivity matrix satisfies

$$
\begin{aligned}
& \left\|S_{0}(\mathrm{i} \omega)\right\| \leqslant \varepsilon \\
& \quad \text { for all real } \omega \text { with }|\omega| \leqslant \omega_{0}, \\
& \left\|S_{0}(s)-\left(I+\frac{\rho}{s} M\right)^{-1}\right\| \dot{\leqslant} \varepsilon \\
& \quad \text { for all }|s| \geqslant \omega_{0} \text { with } \operatorname{Re}(s) \geqslant 0 .
\end{aligned}
$$

Here $M$ is any $p \times p$ real matrix with all its characteristic values in the open right-half complex plane.

Proof. The proof is based on well-known properties of the linear optimal regulator problem (see e.g. [9]). We represent the nominal plant by the state equations

$\dot{x}(t)=A x(t)+B u(t), \quad z(t)=D x(t)$.

and consider controllers that are the interconnection of a full-order observer

$\dot{\hat{x}}(t)=A \hat{x}(t)+B u(t)+K[z(t)-D \hat{x}(t)]$

with a state feedback law $u(t)=-F \hat{x}(t)$. The feedback gain $F$ is chosen as $F_{a}:=\sigma B^{\mathrm{T}} P_{a}$, where for each $\sigma$ the matrix $P_{\sigma}$ is the unique nonnegative-definite symmetric solution of the algebraich Riccati equation

$0=A^{\top} P_{\sigma}+P_{\sigma} A+D^{\top} D-\sigma^{2} P_{\sigma} B B^{\top} P_{\sigma}$.

Here $\sigma$ is a positive scalar parameter. It is well known $[9,10]$ that under the assumptions of invertibility of the plant and location of its transmission zeros in the left-half plane, the matrix $P_{o}$ approaches the zero matrix as $\sigma \rightarrow \infty$. It then follows from the algebraic Riccati equation that as $\sigma \rightarrow \infty$, the gain $F_{\sigma}$ behaves as $\sigma \Gamma_{\sigma} D$, where $\Gamma_{\sigma}$ is some orthogonal matrix, in general dependent on $\sigma$. This is to be understood in the sense that

$$
\lim _{a \rightarrow \infty} \sigma^{-1} \Gamma_{a}^{-1} F_{0}=D \text {. }
$$

In the observer/state feedback arrangement the controller transfer matrix is

$$
\begin{aligned}
G(s)= & {\left[I+F_{\mathrm{o}}(s I-A+K D)^{-1} B\right]^{-1} } \\
& \cdot F_{\mathrm{o}}(s I-A+K D)^{-1} K .
\end{aligned}
$$

This shows that as $\sigma \rightarrow \infty$ the controller transfer matrix approaches

$$
\begin{aligned}
\bar{G}(s):= & {\left[D(s I-A+K D)^{-1} B\right]^{-1} } \\
& \cdot D(s I-A+K D)^{-1} K,
\end{aligned}
$$

which may be rewritten as

$$
\bar{G}(s)=\left[D(s I-A)^{-1} B\right]^{-1} D(s I-A)^{-1} K .
$$

Consequently, the sensitivity matrix $S_{0}(s)$ of the closed-loop system approaches

$$
\begin{aligned}
\bar{S}(s): & =\left[I+H_{0}(s) \bar{G}(s)\right]^{-1} \\
& =\left[I+D(s I-A)^{-1} K\right]^{-1} .
\end{aligned}
$$

Since the closed-loop poles consist of the regulator poles (i.e. the eigenvalues of $A-B F_{\mathrm{o}}$ ), which by construction are in the open left-half complex plane, together with the observer poles (i.e. the eigenvalues of $A-K D$ ), which will also be placed in the open left-half complex plane, the convergence of $S_{0}(s)$ to $\bar{S}(s)$ is uniform in the closed right-half complex plane.

We next choose the observer gain $K$ as $K_{\rho}:=$ $\rho \hat{B}(\mathrm{D} \hat{B})^{-1} M$. Here $\rho$ is a positive scalar parameter. $\hat{B}$ is a constant matrix such that the system

$\dot{x}(t)=A x(t)+\hat{B} v(t), \quad z(t)=D x(t)$

has the same number of inputs as outputs $p$, is stabilizable and detectable and possesses $n-p$ transmission zeros in the open left-half complex plane. These assumptions imply that $D \hat{B}$ is square and nonsingular. Finally, $M$ is the matrix occurring in the theorem statement; it is square and has all its eigenvalues in the open left-half complex plane. As $\rho \rightarrow \infty, n-p$ of the observer poles approach the transmission zeros of

$\dot{x}(t)=A x(t)+\hat{B} v(t), \quad z(t)=D x(t)$,

while the remaining observer poles behave as the roots of $\operatorname{det}(s I+\rho M)$. By the assumptions on the transmission zeros and on the eigenvalues of $M$ the observer is stable for $\rho$ large enough. The corresponding behavior of the asymptotic sensitivity matrix $\bar{S}$ is

$\bar{S}(s)=\left[I+\rho D(s I-A)^{-1} \hat{B}(D \hat{B})^{-1} M\right]^{-1}$

This shows that when $\omega$ is real, by choosing $\rho$ large enough $\bar{S}(i \omega)$ can be made uniformly small for 
$|\omega| \leqslant \omega_{0}$, where $\omega_{0}$ is any positive number. On the other hand, if $\omega_{0}$ is sufficiently large, $\bar{S}(s)$ can be approximated by

$\left(I+\frac{\rho}{s} M\right)^{-1}$

for $|s| \geqslant \omega_{0}$.

\section{A condition for robust stabilizability}

In this section we show that 'desensitized' control systems as discussed in the preceding section are also robust. Under the assumption that the nominal plant is invertible one may define for each plant in the family op with transfer matrix $H$ the matrix function

$\Pi(s):=H(s) H_{0}^{-1}(s)$,

where $H_{0}$ is the transfer matrix of the nominal plant. Since $H(s)=\Pi(s) H_{0}(s)$, the function $\Pi$ represents a multiplicative perturbation of the plant. We shall assume that for each plant in 9 the limit

$\Pi_{\infty}:=\lim _{|s| \rightarrow \infty} \Pi(s)$

exists.

The main result of the paper is the following.

Theorem 2. Suppose that the family of plants 9 satisfies the following assumptions.

(a) Each plant in $\mathcal{P}$ is a nonsingular perturbation of the nominal plant, has the same number of transmission zeros as the nominal plant, and is stabilizable and detectable.

(b) The transmission zeros of each plant all lie in a bounded region in the open left-half complex plane.

(c) There exists a fixed square matrix $M$ with all its eigenvalues in the open right-half complex plane such that for each plant the matrix $\Pi_{\infty} M$ has all its eigenvalues in a bounded region in the open right-half complex plane.

Then there exists a single controller that stabilizes the control system for each plant in the family $\mathcal{P}$.

Proof. We consider controllers that are constructed as in the proof of Theorem 1, with the parameters $\sigma$ and $\rho$ sufficiently large. We first recall that in the configuration of Figure 1 , the characteristic polynomial of the closed-loop sys- tem $\phi_{c}$ is given by

$\phi_{\mathrm{c}}(s)=\phi(s) \operatorname{det}[I+L(s)]$.

Here $\phi$ is the open-loop characteristic polynomial, i.e. the characteristic polynomial of the series connection of the controller and the plant. As before, $L$ is the loop gain. Denoting by $L_{0}$ the nominal loop gain we have

$$
\begin{aligned}
L(s) & =H(s) G(s) \\
& =\Pi(s) H_{0}(s) G(s)=\Pi(s) L_{0}(s)
\end{aligned}
$$

and obtain, omitting the argument $s$,

$$
\begin{aligned}
\phi_{c}= & \phi \operatorname{det}\left(I+\Pi L_{0}\right) \\
= & \phi \operatorname{det}\left(I+L_{0}\right) \\
& \times \operatorname{det}\left[\left(I+L_{0}\right)^{-1}+\Pi L_{0}\left(I+L_{0}\right)^{-1}\right] \\
= & \phi \operatorname{det}\left(I+L_{0}\right) \operatorname{det}\left[S_{0}+\Pi\left(I-S_{0}\right)\right] \\
= & \phi \frac{\phi_{c 0}}{\phi_{0}} \operatorname{det}\left[S_{0}+\Pi\left(I-S_{0}\right)\right] .
\end{aligned}
$$

Here $\phi_{\mathrm{c} 0}$ is the nominal closed-loop characteristic polynomial, $\phi_{0}$ the nominal open-loop characteristic polynomial, and $S_{0}$ the nominal sensitivity matrix.

Using Rouche's theorem, we compare the closed-loop characteristic polynomial

$$
\begin{aligned}
\phi_{\mathrm{c}}(s)= & \phi_{\mathrm{c} 0}(s) \frac{\phi(s)}{\phi_{0}(s)} \\
& \times \operatorname{det}\left\{S_{0}(s)+\Pi(s)\left[I-S_{0}(s)\right]\right\}
\end{aligned}
$$

to the rational function

$\phi_{\infty}(s):=\phi_{\mathrm{c} 0}(s) \frac{\psi(s) \operatorname{det}\left(s I+\rho \Pi_{\infty} M\right)}{\psi_{0}(s) \operatorname{det}(s I+\rho M) \operatorname{det}\left(\Pi_{\infty}\right)}$

on a Nyquist contour as indicated in Figure 2. Here $\psi$ is the polynomial defined by

$\psi(s):=\phi_{\mathrm{p}}(s) \operatorname{det}[H(s)]$,

where $\phi_{p}$ is the plant characteristic polynomial. The roots of $\psi$ are the transmission zeros of the plant. Similarly,

$\psi_{0}(s):=\phi_{\mathrm{p} 0}(s) \operatorname{det}\left[H_{0}(s)\right]$,

with $\phi_{p 0}$ the characteristic polynomial of the nominal plant. Since $\phi_{\mathrm{c}}$ is a polynomial, it is analytic on and inside the contour. By the assumptions that 


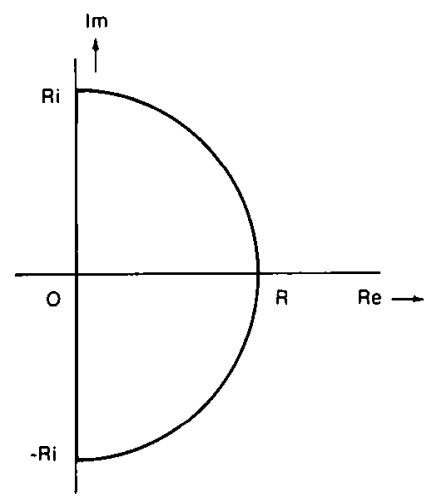

Fig. 2. Nyquist contour, consisting of part of the imaginary axis and a semicircle with radius $R$.

the nominal transmission zeros are in the left-half complex plane and that $M$ has all its eigenvalues in the open right-half complex plane, the rational function $\phi_{\infty}$ is also analytic on and inside the Nyquist contour. Rouché's theorem then states that if

$$
\left|\frac{\phi_{c}(s)-\phi_{\infty}(s)}{\phi_{\infty}(s)}\right|<1
$$

on the Nyquist contour, $\phi_{c}$ and $\phi_{\infty}$ have the same number of zeros inside the contour. The inequality (14) may be proved by taking $\varepsilon$ small enough and $\omega_{0}$ large enough, and using the approximation (1) on the vertical part of the contour for $|\omega| \leqslant \omega_{0}<R$ and the approximation (2) on the remaining part of the contour. Since the nominal closed-loop system is stable, and by the assumptions both the polynomial $\psi$ and $\operatorname{det}\left(s I+\rho \Pi_{\infty} M\right)$ have their roots in the open left-half complex plane, $\phi_{\infty}$ and hence also $\phi_{\mathrm{c}}$ has no roots inside the contour. Since this holds for any $R$ that is large enough, we conclude that $\phi_{c}$ has no roots in the right-half complex plane and hence the closed-loop system is stable.

\section{Discussion}

Theorem 2 shows that robust stabilization can be obtained for square linear time-invariant finite-dimensional plants, provided that under perturbation all transmission zeros remain in the lefthalf complex plane and moreover a condition on the gain at infinite frequency is satisfied.

In the single-input single-output case the in- finite-frequency condition takes a simpler form. Suppose that the transfer function of the plant is expressed as

$H(s)=k \chi(s) / \phi_{\mathrm{p}}(s)$,

where $\phi_{\mathrm{p}}$ is the plant characteristic polynomial, $\chi \mathrm{a}$ monic polynomial and $k$ a scalar constant. Then the infinite-frequency condition reduces to the requirement that for each plant in the family $Q 9$ the constant $k$ has the same sign.

In the multi-input multi-output case a sufficient but not necessary condition for the high-frequency requirement is that for each plant the matrix $\Pi_{\infty}$ has all its eigenvalues in the open right-half complex plane; the high-frequency condition then is met with $M=I$.

Central in the conditions for the existence of a single stabilizing controller is the requirement that under perturbation the transmission zeros of the plant remain in the left-half complex plane. The condition of right-half plane transmission zeros appears very often in problems of control design and therefore is quite natural. It emerges for instance, together with the infinite-frequency condition as specialized to the single-input single-output case, in investigations of the stability of adaptive control schemes (see Morse [12] and Narendra, Lin and Valavani [13]). This points to an interesting connection between adaptive and robust control.

It is clear that there are instances where transmission zeros cross over to the right-half complex plane due to plant perturbations. just as there are problems where there always are transmission zeros in the right-half complex plane. In such situations there are essential bounds on what can be achieved by feedback (see e.g. Zames [7]) and it may well be that there is no single controller that stabilizes all plants in the family $\mathscr{P}$.

It is of interest to note that the theorem applies to families of plants whose members may all or in part be open-loop unstable. There is no veto on perturbations that make open-loop poles cross over into or out of the right-half complex plane. On the other hand, the theorem is restricted to plants that are nonsingular perturbations of a nominal plant and that also retain all their transmission zeros. It remains to be investigated whether the result can be extended to the interesting and practically important case of singular perturbations. 
Since the proof of the existence of a robustly stabilizing controller is constructive, it is in principle clear how to design such controllers. It is still a partly open question, however, how to design robustly stabilizing controllers that at the same time satisfy other design objectives such as compatibility with plant capacity and good response properties.

\section{References}

[1] W.M. Wonham, Linear Multivariable Control: A Geometric Approach (Springer, New York, 1979).

[2] E.J. Davison and I.J. Ferguson, The design of controllers for the multi-variable robust servomechanism problem using parameter optimization methods, IEEE Trans. Aut. Control 26 (1981) 93-110.

[3] $M$. Safonov and $M$. Athans, Gain and phase margins for multiloop LQG regulators, IEEE Trans. Aut. Control 22 (1977) 173- 179.

[4] J.C. Doyle, Robustness of multiloop linear feedback systems, Proc. IEEE Conf. on Decision and Control, San Diego (Jan. 1978).
[5] J.C. Doyle and G. Stein. Multivariable feedback design: Concepts for a classical/modern synthesis, IEEE Trans. Aut. Control 26 (1981) 4-16.

[6] R. Saeks and J. Murray, Fractional representation, algebraic geometry, and the simultaneous stabilization problem. Proc, 1981 IEEE International Symposium on Circuits and Systems. Chicago, IL (April 27-29, 1981) 463-464.

[7] G. Zames, Feedback and optimal sensitivity: Model reference transformations, multiplicative seminorms, and approximate inverses, IEEE Trans. Aut. Control 26 (1981) $301-320$.

[8] H. Kimura, Perfect and subperfect regulation in linear multivariable control systems, Automatica 17 (1981).

[9] H. Kwakernaak and R. Sivan, Linear Optimal Control Systems (Wiley-Interscience, New York, 1972).

[10] H. Kwakernaak and R. Sivan, The maximally achievable accuracy of linear optimal regulators and filters, IEEE Trans. Aut. Control 17 (1972) 79-86.

[11] B.A. Francis, The optimal linear quadratic time-invariant regulator with cheap control, IEEE Trans. Aut. Control. 24 (1979) 616-621.

[12] A.S. Morse, Global stability of parameter-adaptive control systems, IEEE Trans. Aut. Control 25 (1980) 433-439.

[13] K.S. Narendra, Y.-H. Lin and L.S. Valavani, Stable adaplive controller design. Part II: Proof of stability, IEEE Trans. Aut. Control 25 (1980) 440-448. 